\title{
Corrigendum to "Structural Stability, Transitions, and Interactions within SoxYZCD-Thiosulphate from Sulfurimonas denitrificans: An In Silico Molecular Outlook for Maintaining Environmental Sulphur Cycle"
}

\author{
Sujay Ray ${ }^{1}$ and Arundhati Banerjee ${ }^{2}$ \\ ${ }^{1}$ Department of Biochemistry and Biophysics, University of Kalyani, Kalyani, Nadia, India \\ ${ }^{2}$ Department of Biotechnology, National Institute of Technology, Mahatma Gandhi Avenue, Durgapur, West Bengal, India \\ Correspondence should be addressed to Sujay Ray; raysujay@gmail.com
}

Received 2 November 2016; Accepted 13 November 2016

Copyright (C) 2016 S. Ray and A. Banerjee. This is an open access article distributed under the Creative Commons Attribution License, which permits unrestricted use, distribution, and reproduction in any medium, provided the original work is properly cited.

In the article titled "Structural Stability, Transitions, and Interactions within SoxYZCD-Thiosulphate from Sulfurimonas denitrificans: An In Silico Molecular Outlook for Maintaining Environmental Sulphur Cycle" [1], we found three sentences that need to be modified in the third section: Results and Discussion. In Section 3.1, the sentence "The modeled SoxC structure is well illustrated in Figure 1 with red, cyan, and magenta shades representing $\beta$-sheets, $\alpha$-helices, and interspersing coils, respectively" should be changed to "The modeled SoxC structure is well illustrated in magenta shades in Figure 1 with prominent secondary structure conformations ( $\beta$-sheets, $\alpha$-helices, and interspersing coils)." In Section 3.2, the sentence "The entire protein was interspersed with coil regions. SoxD structure is illustrated in Figure 1 with $\alpha$-helices and coils in yellow and pink shades, respectively" should be changed to "The entire protein was interspersed with coil regions. SoxD structure is illustrated in Figure 1 with yellow shades showing the prominent secondary structures possessing $\alpha$-helices and coils." Finally, the sentence "The pictorial representation with tabulation of the conformational switches is illustrated in Figures 4 and 5, respectively, for SoxY and SoxZ proteins" should be changed to "The pictorial representation with tabulation of the conformational switches is illustrated in Figures 3 and 4, respectively, for SoxY and SoxZ proteins" in Section 3.6.2.

\section{References}

[1] S. Ray and A. Banerjee, "Structural stability, transitions, and interactions within SoxYZCD-thiosulphate from Sulfurimonas denitrificans: an in silico molecular outlook for maintaining environmental sulphur cycle," Journal of Biophysics, vol. 2016, Article ID 8683713, 10 pages, 2016. 

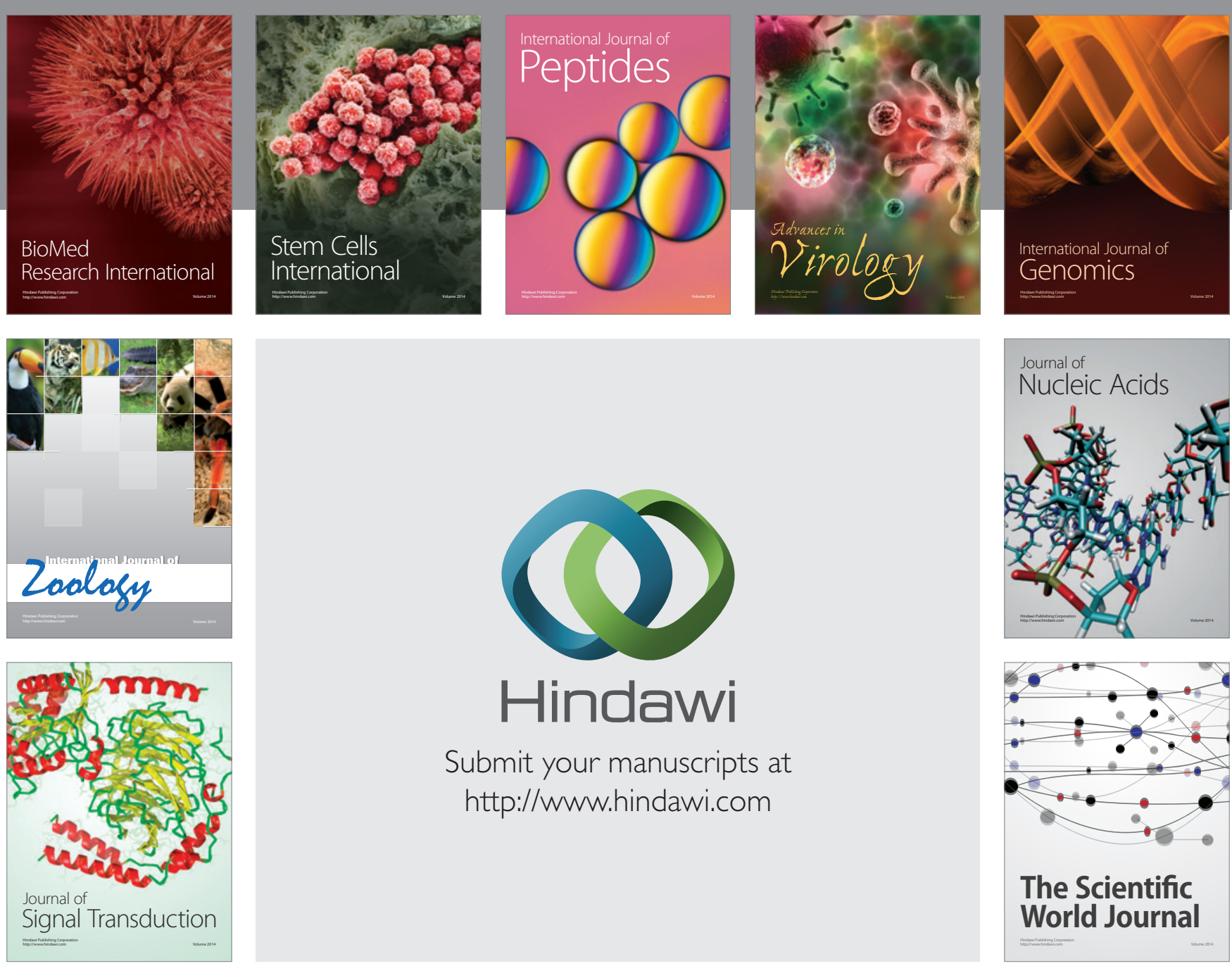

Submit your manuscripts at

http://www.hindawi.com
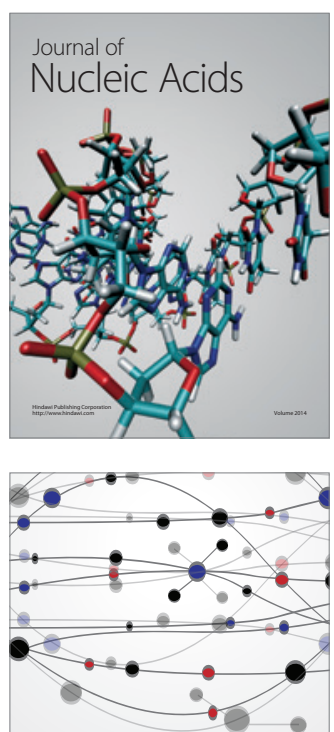

The Scientific World Journal
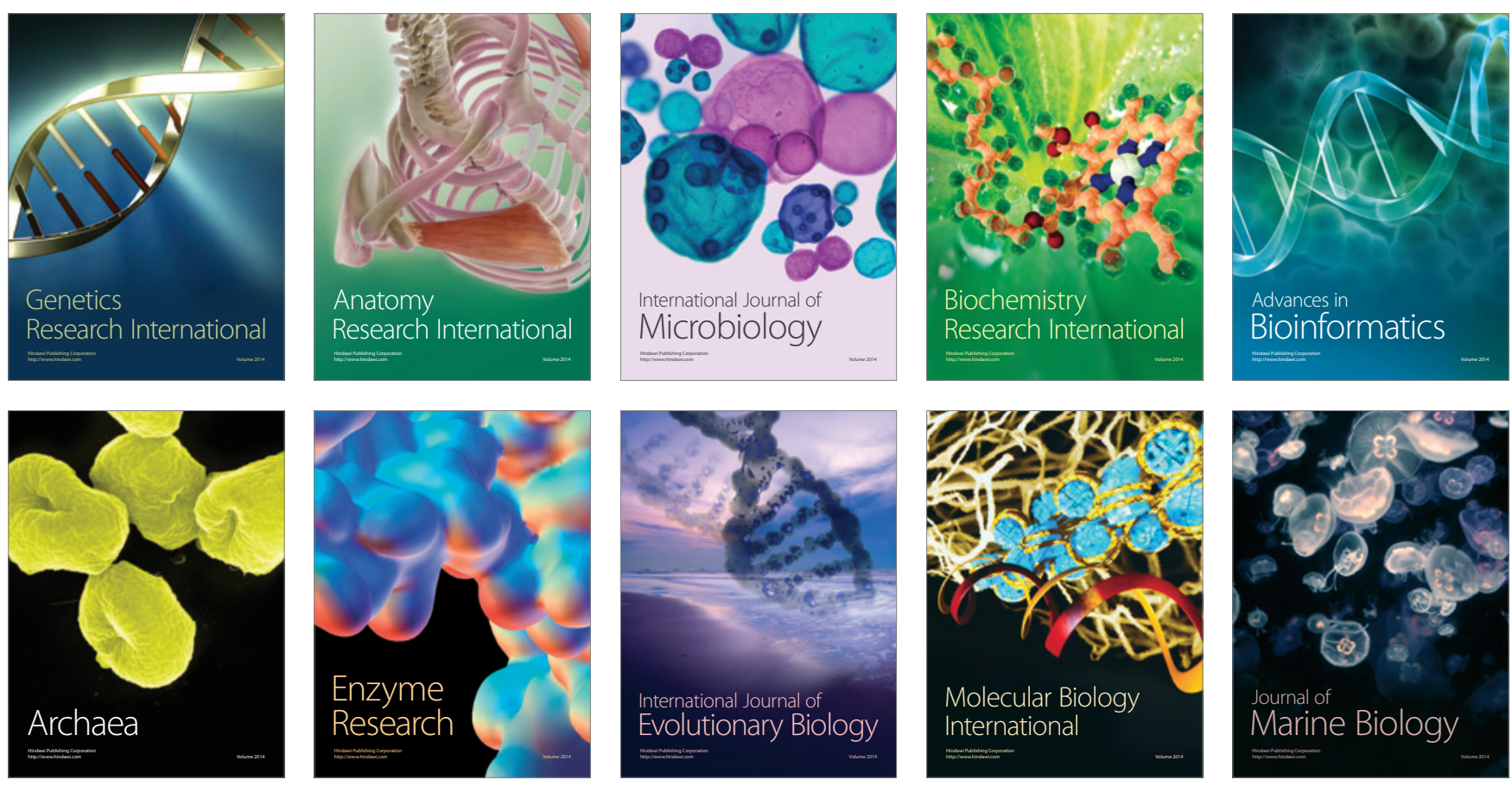